\title{
Immediate and Sustained Improvement in Behavior and Life Quality by Adenotonsillectomy in Children With Sleep-Disordered Breathing
}

\begin{abstract}
Yung Jin Jeon ${ }^{1, \star} \cdot$ Jae-Jin Song ${ }^{2, \star} \cdot$ Jae-Cheul Ahn ${ }^{3}$. I Gyu Kong ${ }^{4} \cdot$ Jae-Won Kim $^{5} \cdot$ Gyeong-Hun Park $^{6} \cdot$ Tae-Bin Won $^{1}$
${ }^{1}$ Department of Otorhinolaryngology-Head and Neck Surgery, Seoul National University Hospital, Seoul; ${ }^{2}$ Department of OtorhinolaryngologyHead and Neck Surgery, Seoul National University Bundang Hospital, Seongnam; ${ }^{3}$ Department of Otolaryngology-Head and Neck Surgery, CHA Bundang Medical Center, CHA University, Seongnam; ${ }^{4}$ Department of Otorhinolaryngology-Head and Neck Surgery, Hallym University Sacred Heart Hospital, Anyang; ${ }^{5}$ Division of Child and Adolescent Psychiatry, Department of Psychiatry, Seoul National University College of Medicine, Seoul; ${ }^{6}$ Department of Dermatology, Hallym University Dongtan Sacred Heart Hospital, Hallym University College of Medicine, Hwaseong,
\end{abstract}

Objectives. To investigate longitudinal changes in behavior and quality-of-life (QoL) in children with sleep disordered breathing (SDB) after adenotonsillectomy (AT).

Methods. This study prospectively enrolled 148 children who underwent AT for SDB. Caregivers filled out Korean attention deficit hyperactivity disorder (ADHD) rating scale (K-ARS) and Korean version of the obstructive sleep apnea-18 (KOSA-18) at preoperative 1 day, postoperative 1 month, and postoperative 6 months. Longitudinal changes in KARS and KOSA-18 were evaluated and compared among subgroups.

Results. Both K-ARS and KOSA-18 scores improved immediately at postoperative 1 month and were maintained at postoperative 6 months. The mean preoperative K-ARS scores of the non-ADHD, ADHD-trait, and overt-ADHD groups showed uniformly immediate and sustained improvements. Also, the mean preoperative KOSA-18 scores of the small, moderate, and large impact groups were significantly improved both at postoperative 1 month and 6 months. The changes in behavior and QoL showed no significant differences with regard to the aforementioned factors.

Conclusion. After AT, we may expect immediate and sustained improvements in behavior and QoL in children with SDB regardless of preoperative symptom severity. Moreover, by performing AT, improvements in behavior and QoL are expected regardless of sex, age, allergic trait, or concurrent coblation-assisted turbinoplasty. These findings may help our colleague physicians counselling pediatric SDB patients and their caregivers.

Keywords. Sleep Apnea Syndromes; Attention Deficit Disorder with Hyperactivity; Quality of Life; Tonsillectomy; Adenoidectomy

\footnotetext{
- Received April 13, 2015

Revised May 9, 2015

Accepted May 26, 2015

- Corresponding author: Tae-Bin Won

Department of Otorhinolaryngology-Head and Neck Surgery, Seoul

National University Hospital, 101 Daehak-ro, Jongno-gu, Seoul 03080,

Korea

Tel: +82-2-2072-4037, Fax: +82-2-745-2387

E-mail: binent@hanmail.net

- The first two authors contributed equally to this study.
}

\section{INTRODUCTION}

Sleep disordered breathing (SDB) is common in children, affecting approximately $11 \%-40 \%$ of the pediatric population [1]. Pediatric SDB has a unique and significant impact on daytime functioning and development not seen in adult SDB, including learning difficulty, growth retardation, hyperactivity and inattention, and poor emotional regulation [2].

It has been suggested that proper treatment of SDB may improve attention deficit hyperactivity disorder (ADHD) in a subset of children [3]. Considering that adenotonsillectomy (AT) is

Copyright $\odot 2016$ by Korean Society of Otorhinolaryngology-Head and Neck Surgery.

This is an open-access article distributed under the terms of the Creative Commons Attribution Non-Commercial License (http://creativecommons.org/licenses/by-nc/4.0)

which permits unrestricted non-commercial use, distribution, and reproduction in any medium, provided the original work is properly cited. 
the most effective treatment modality that leads to significant improvements in pediatric SDB [4]. AT may be able to relieve SBD-related behavioral problems. Indeed, previous studies have demonstrated that AT results in marked improvement in scales regarding neurocognitive problems or inattention, hyperactivity, and ADHD [5]. Also, alternative scales, such as quality-of-life (QoL) measures, have indicated that AT results in marked improvement in those clinically diagnosed as having SDB [1].

Although these previous works have elucidated the effectiveness of AT in treating pediatric SDB patients with behavioral problems or with decrement in SBD-related QoL, only a few studies have investigated postoperative improvements at both short- and long-term follow-up periods [6,7]. Also, only a few studies have evaluated other combined factors, such as allergic rhinitis [8], that may contribute to residual symptoms and signs of SDB after AT. Additionally, no study, to our knowledge, has studied and reported on all of these variables in the same cohort of patients.

Hence, the purpose of the current study was 2 folds. First, by means of serial (preoperative 1 day, postoperative 1 month, and postoperative 6 months) questionnaires on ADHD and sleep disorder-specific QoL, we sought to reveal longitudinal changes in behavior and QoL in a prospective pediatric cohort with SDB after AT. Second, by subdividing the cohort according to sex, age group, allergic trait, and concurrent coblation-assisted turbinoplasty (CAT), we investigated possible factors that may affect the surgical outcome regarding changes in behavior and QoL.

\section{MATERIALS AND METHODS}

\section{Patients}

The subjects who were clinically diagnosed as having SDB and were planned for AT at Seoul National University Hospital from January 2011 to June 2014 were recruited prospectively. The mean age of the subjects was $7.3 \pm 2.6$ years old (range, 3-13 years). This study was reviewed and approved by the Institutional Review Board of the Clinical Research Institute at Seoul National University Hospital and the procedures followed were in accordance with the Helsinki Declaration of 1975. Caregivers of a total of 148 subjects were requested to complete questionnaires at preoperative 1 day, postoperative 1 month, and postoperative 6 months. Of 148 patients who completed preoperative 1 day and postoperative 1 month questionnaires, 72 patients completed follow-up questionnaires at postoperative 6 months. Syndromic children with craniofacial anomaly were excluded from the study. The subjects' allergic trait was assessed by skinprick test or serum multiple allergen simultaneous test, and patients who displayed positive results on these tests with allergic symptoms were regarded to have allergic trait. Concurrent CAT was performed together with AT if the patient showed signs of inferior turbinate hypertrophy with or without allergic trait.

\section{Questionnaires}

Korean ADHD rating scale (K-ARS) and Korean version of the obstructive sleep apnea-18 (KOSA-18) were used for this study. The K-ARS is a validated Korean-translated version [9] of the ADHD rating scale-IV [10]. This scale consists of 18 items with 2 subscales (9 for inattention domain and 9 for hyperactivityimpulsivity domain, each of which is scored independently) originating from Diagnostic and Statistical Manual of Mental Disorders - 4th Edition (DSM-IV) ADHD diagnostic criteria, as its original version. Based on previous literature, subjects with a K-ARS score less than 18 were regarded as "non-ADHD," between 19 and 28 as "ADHD trait," and more than 28 as "overt ADHD" [11].

Meanwhile, the KOSA-18, a Korean version [12] of obstructive sleep apnea-18 scores [13], is an index to evaluate the QoL impact with regard to physical and emotional problems for obstructive sleep apnea syndrome (OSAS) in chidren. KOSA-18 scores less than 60 suggest a small impact, scores between 60 and 80 a moderate impact, and scores above 80 a large impact on the QoL [14].

\section{Statistical analyses}

Longitudinal changes in K-ARS and KOSA-18 scores of the subjects were evaluated by the paired $t$-test/Wilcoxon signed rank test, while comparisons between follow-up completed subjects and follow-up lost subjects at a certain time point were performed by the independent $t$-test. Group comparisons with regard to the longitudinal changes in K-ARS and KOSA-18 scores at each time point were performed by the repeated measures analysis of variance (RM-ANOVA)/Kruskal-Wallis test. Statistical analyses were performed using IBM SPSS Statistics ver. 20.0 (IBM Co., Armonk, NY, USA). The criterion for statistical significance was set at $P<0.05$.

\section{RESULTS}

\section{Longitudinal changes in K-ARS and KOSA-18 scores}

The mean preoperative K-ARS score of the 148 patients surveyed at preoperative 1 day was $12.5 \pm 9.7$, which improved to $7.0 \pm 6.4$ at postoperative 1 month $(P<0.01$, paired $t$-test). The mean K-ARS score of the 72 patients who completed the 6-month follow-up was $8.4 \pm 7.7$, which was still significantly lower than the preoperative mean score $(P<0.01$, paired $t$-test). The mean K-ARS scores of the 72 patients at postoperative 1 month (6.2 \pm 5.4$)$ was not significantly different from that at 6 months ( $P=0.13$, paired $t$-test), and the mean K-ARS score of the 76 follow-up lost patients at 1 month $(6.6 \pm 6.3)$ was not different from that of the 72 follow-up completed patients $(P=$ 0.46 , independent $t$-test). When divided into subscales, the mean preoperative attention deficit domain and hyperactivity-impulsivity domain scores $(6.2 \pm 5.3$ and $6.2 \pm 5.1$, respectively) de- 

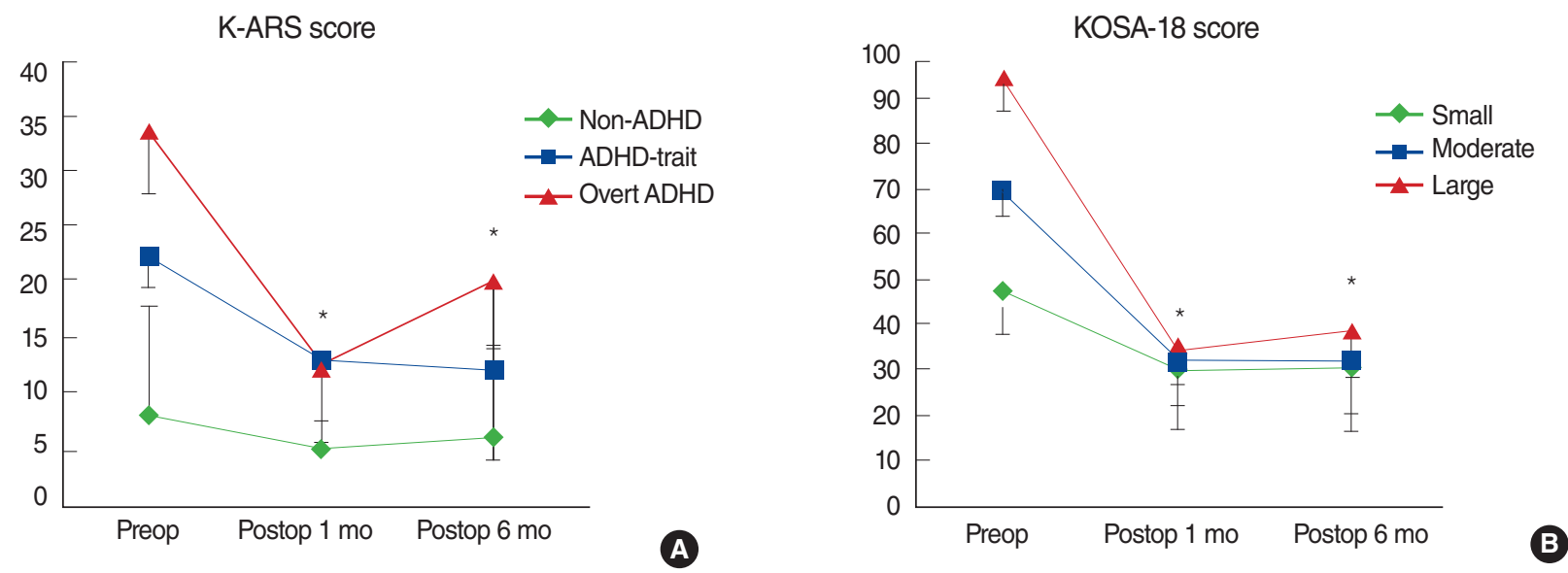

Fig. 1. Comparison of longitudinal changes in Korean attention deficit hyperactivity disorder (ADHD) rating scale (K-ARS; $A$ ) and Korean version of the obstructive sleep apnea-18 (KOSA-18; B) scores. Postop, postoperative. ${ }^{*} P<0.05$, statistically significant differences among groups.

creased significantly at postoperative 1 month $(3.1 \pm 3.2$ and $3.9 \pm 3.6$, respectively) and at 6 months $(4.1 \pm 3.8$ and $4.7 \pm 4.7$, respectively) (all $P<0.01$, paired $t$-test).

Meanwhile, the mean KOSA-18 scores at postoperative 1 month $(32.2 \pm 10.4)$ and at 6 months $(32.5 \pm 11.6)$ were significantly lower than the preoperative 1 day score $(68.5 \pm 19.9)$ (both $P<0.01$, paired $t$-test), but the means of the 72 follow-up completed patients at postoperative 1 month and 6 months were not significantly different $(31.9 \pm 8.3$ and $32.5 \pm 11.6$, $P=0.68$ by the paired $t$-test). The mean KOSA- 18 score of the 76 follow-up lost patients at 1 month $(32.4 \pm 12.1)$ was not different from that of the 72 follow-up completed patients $(P=$ 0.76 , independent $t$-test).

\section{Comparison of longitudinal changes in K-ARS scores among the overt ADHD, ADHD-trait, and non-ADHD groups}

Based on preoperative K-ARS scores, 13 subjects were classified as overt ADHD, 23 as ADHD-trait, and 112 as non-ADHD. At postoperative 1 month, in contrast, only 1 subject remained as overt ADHD and 8 as ADHD-trait while the other 139 were classified as non-ADHD. The mean preoperative K-ARS scores of the non-ADHD, ADHD-trait, and overt ADHD groups (mean \pm standard error [SE]; 8.0 $\pm 5.6,22.2 \pm 2.7$, and 33.4 \pm 9.7 , respectively) decreased significantly both at postoperative 1 month $(5.1 \pm 4.8,13.04 \pm 7.4$, and $12.38 \pm 8.0$, respectively) and 6 months (6.1 $\pm 5.8,12.0 \pm 7.9$, and $20.1 \pm 7.9$, respectively) (all $P<0.001$, paired $t$-test for the overt ADHD group; $P=0.002$ and $P=0.002$, Wilcoxon signed rank test for the ADHD-trait group; $P=0.018$ and $P=0.018$, Wilcoxon signed rank test for the overt ADHD group) (Fig. 1A). Of note, 11 of 13 overt ADHD subjects and 18 of 23 ADHD-trait subjects became no-ADHD at postoperative 1 month. Also, the mean K-ARS scores of the overt ADHD and ADHD-trait groups decreased to the level of "nonADHD" $(<19)$. However, the overt ADHD group and the AD-
HD-trait group showed significantly higher mean K-ARS scores than the non-ADHD group at postoperative 1 month $(P<0.01$, Kruskal-Wallis test). Additionally, the overt ADHD group displayed significantly increased mean K-ARS score at postoperative 6 months as compared with that at postoperative 1 month $(P=0.028$, Wilcoxon signed rank test).

\section{Comparison of longitudinal changes in KOSA-18 scores among the small, moderate, and large impact groups} Based on preoperative KOSA-18 scores, 55 subjects were allocated to the small impact group, 55 to the moderate impact group, and 38 to the large impact group. The mean preoperative KOSA-18 scores of the small, moderate, and large impact groups (mean \pm SE; 48.0 $\pm 7.3,70.6 \pm 5.3$, and $95.3 \pm 9.6$, respectively) were significantly improved both at postoperative 1 month (30.3 $\pm 8.0,32.3 \pm 10.0$, and $34.8 \pm 13.4$, respectively) and 6 months (30.6 $\pm 10.2,32.3 \pm 11.8$, and $38.8 \pm 13.8$, respectively) (all $P<0.01$, paired $t$-test for the small and moderate impact groups and Wilcoxon signed rank test for the large impact group) (Fig. 1B). In particular, all but 1 patient who were in either the moderate impact group or the large impact group were reallocated to the small impact group at postoperative 1 month. Also, an ANOVA at postoperative 1 month revealed no statistically significant differences among the 3 groups $(P=0.130)$.

\section{Comparison of longitudinal changes in K-ARS and KOSA-18 scores according to sex}

As illustrated in Fig. 2A, the mean preoperative K-ARS scores of male and female patients (mean \pm SE; $13.7 \pm 9.6$ and 10.6 \pm 9.6 , respectively) improved significantly both at postoperative 1 month (7.8 \pm 6.5 and $5.8 \pm 6.2$, respectively) and 6 months (9.5 \pm 7.9 and $6.6 \pm 7.1$, respectively) (all $P<0.01$, paired $t$-test) (Fig. 2A). However, the interaction effect (K-ARS score $\times$ sex) measured by RM-ANOVA revealed no significant result $(F=$ 

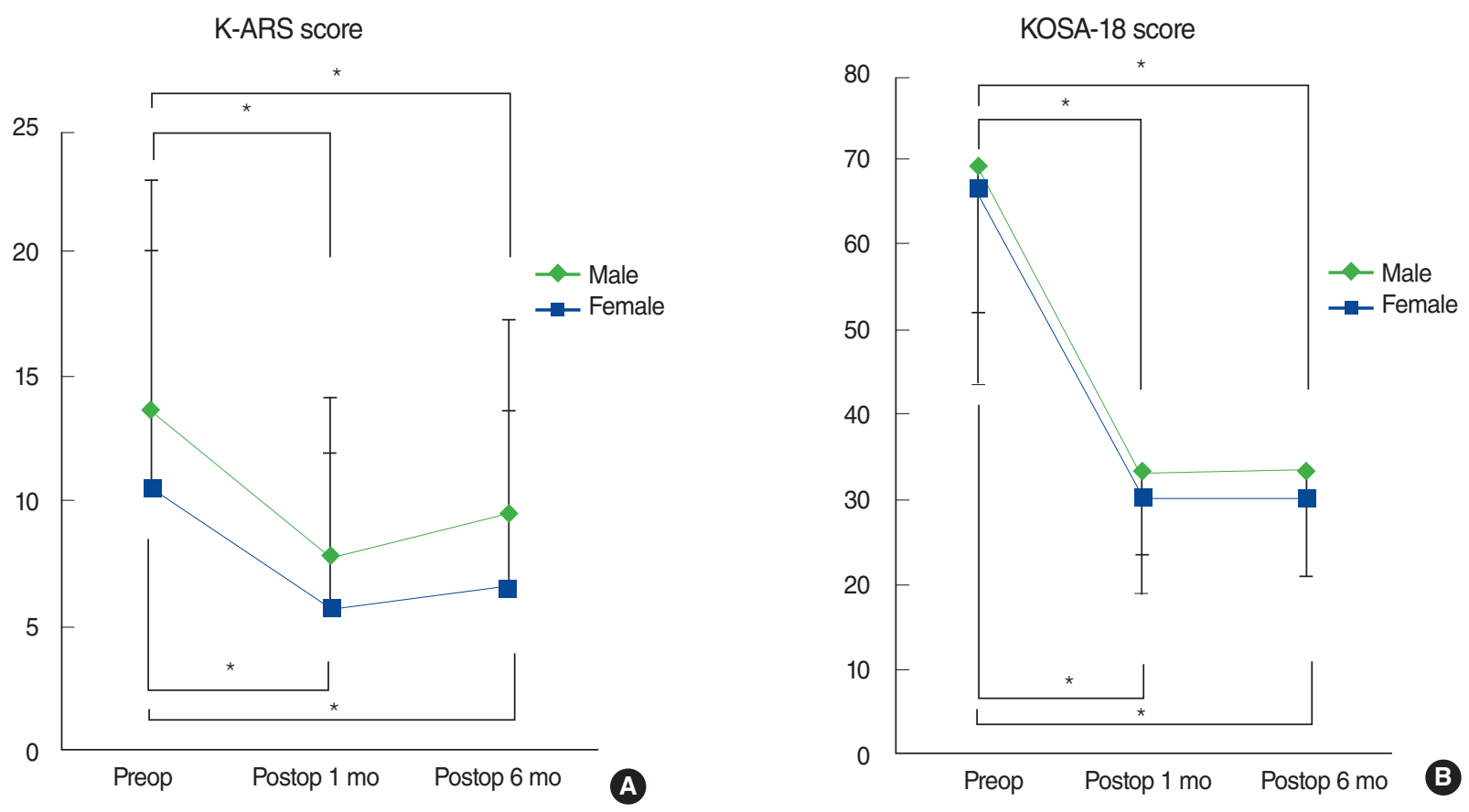

Fig. 2. Longitudinal changes in Korean attention deficit hyperactivity disorder (ADHD) rating scale (K-ARS; A) and Korean version of the obstructive sleep apnea-18 (KOSA-18; B) scores between males and females. ${ }^{*} P<0.05$, statistically significant differences among groups.
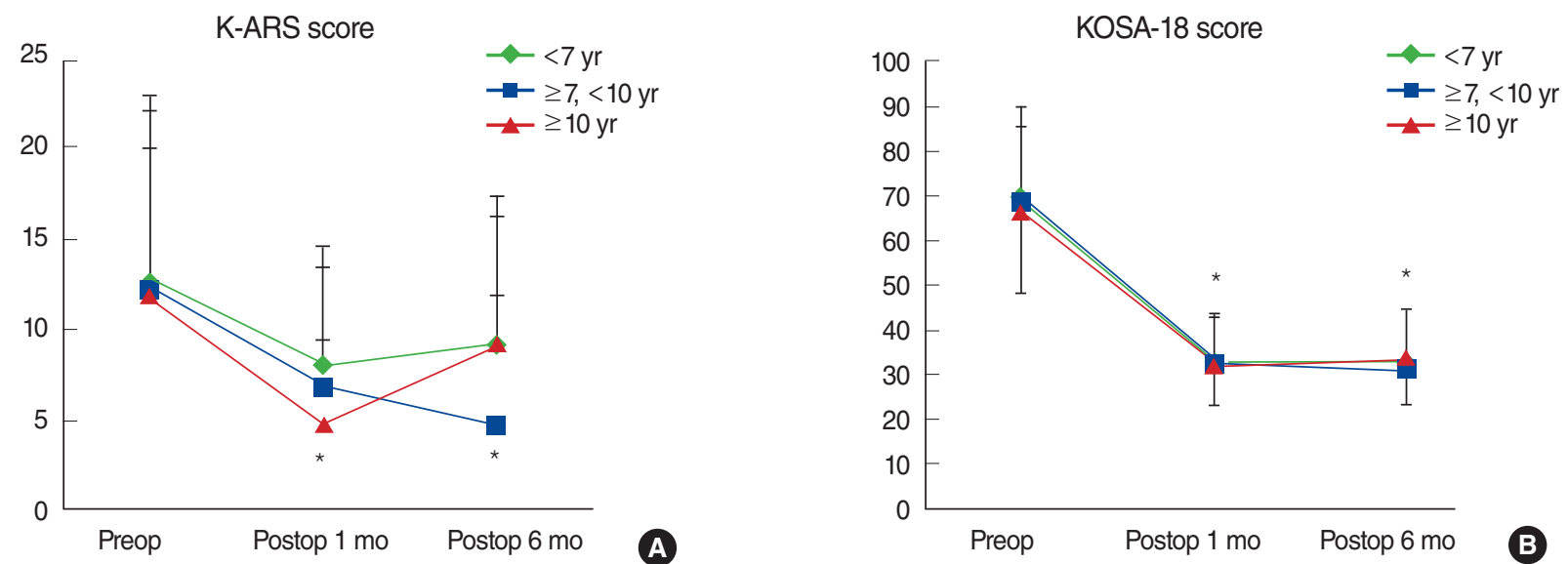

Fig. 3. Longitudinal changes in Korean attention deficit hyperactivity disorder rating scale (K-ARS; $A)$ and Korean version of the obstructive sleep apnea-18 (KOSA-18; B) scores in different age groups: group 1, age <7 years; group 2, $\geq 7,<10$ years; and group 3, age $\geq 10$ years. ${ }^{*} P<0.05$, statistically significant differences among groups.

4.58, $P=0.13)$. Likewise, the mean preoperative KOSA-18 scores of male and female patients (mean \pm SE; $69.3 \pm 17.2$ and $67.2 \pm 23.6$, respectively) decreased significantly both at postoperative 1 month (33.2 \pm 9.6 and $30.6 \pm 11.5$, respectively) and 6 months (33.7 \pm 12.7 and $30.3 \pm 9.2$, respectively) (all $P<0.01$, paired $t$-test). RM-ANOVA on the interaction effect (KOSA-18 score $\times$ sex $)$ showed no significant difference between the male and female group $(F=0.60, P=0.55)$ (Fig. $2 \mathrm{~B})$. In short, both the male and female groups showed significant improvement at all time points as compared with preoperative questionnaire scores, but there were no differences between the 2 groups.
Comparison of longitudinal changes in K-ARS and KOSA-18 scores according to age group

To evaluate possible differences according to age group, the patients were subdivided into 3 age groups: group $1,<7$ years; group $2, \geq 7,<10$ years; and group $3, \geq 10$ years. The age group division was based on school age (preschool age, the lower and upper grade elementary school age). As demonstrated in Fig. $3 \mathrm{~A}$, the mean preoperative K-ARS scores of groups 1,2 , and 3 (mean \pm SD; $12.8 \pm 10.3,12.3 \pm 9.8$, and $12.0 \pm 8.0$, respectively) decreased significantly both at postoperative 1 month $(7.9 \pm 6.7$, $6.9 \pm 6.6$, and $4.9 \pm 5.0$, respectively) and 6 months (9.1 \pm 8.3 , 
6.9 \pm 7.1, and 9.3 \pm 7.0 , respectively) (all $P<0.01$, paired $t$-test) (Fig. 3A). However, the interaction effect (K-ARS score $\times$ age group) measured by RM-ANOVA displayed no significant differences among the 3 groups $(F=2.20, P=0.74)$. Meanwhile, the mean preoperative KOSA-18 scores of groups 1,2 , and 3 (mean \pm SD; $69.5 \pm 18.9,68.4 \pm 21.1$, and $66.0 \pm 21.3$, respectively) decreased significantly both at postoperative 1 month $(32.00 \pm 10.9,32.6 \pm 10.8$, and $32.0 \pm 8.5$, respectively) and 6 months (33.0 $\pm 10.3,31.5 \pm 9.8$, and $33.0 \pm 9.4$, respectively) (all $P<0.01$, paired $t$-test). However, again, RM-ANOVA for the interaction effect (KOSA-18 score $\times$ age group) showed no significant difference between the male and female group $(F=0.77$, $P=0.56$ ) (Fig. 3B). In summary, all 3 age groups showed uniformly significant improvement at all time points as compared with preoperative questionnaire scores, but there were no differences among the 3 groups.

\section{Comparison of longitudinal changes in K-ARS and KOSA-18 scores according to allergic trait}

The mean preoperative K-ARS scores of the allergic trait group and the no allergic trait group (mean \pm SD; $12.2 \pm 9.4$ and $12.7 \pm 10.0$, respectively) improved significantly both at postoperative 1 month (5.9 \pm 5.6 and $7.5 \pm 6.9$, respectively) and 6 months (7.1 \pm 7.8 and $9.3 \pm 7.7$, respectively) (all $P<0.01$, paired $t$-test). Also, the mean preoperative KOSA-18 scores of the 2 groups (mean $\pm \mathrm{SD} ; 69.3 \pm 19.5$ and $68.4 \pm 20.8$, respectively) decreased significantly both at postoperative 1 month (32.7 \pm 10.7 and $32.1 \pm 10.4$, respectively) and at postoperative 6 months (32.4 \pm 13.9 and $33.1 \pm 10.0$, respectively) (all $P<0.01$, paired $t$-test). However, RM-ANOVAs for the interaction effects (K-ARS $\times$ group and KOSA-18 score $\times$ group) showed no significant difference between the 2 groups $(F=0.26, P=0.77$ and $F=2.24, P=0.11$ ). In short, the patients demonstrated consistently significant improvement in behavior and QoL as compared with preoperative status regardless of allergic trait, but there were no significant differences between the allergic trait group and the no allergic trait group.

\section{Comparison of longitudinal changes in K-ARS and KOSA-18 scores between the AT alone group and the AT with CAT group}

The mean preoperative K-ARS scores of the AT alone group and the AT with CAT group (mean \pm SD; $11.8 \pm 9.3$ and $13.8 \pm 10.4$, respectively) decreased significantly both at postoperative 1 month (6.7 \pm 5.9 and $7.6 \pm 7.4$, respectively) and 6 months (7.8 \pm 7.3 and $10.2 \pm 8.6$, respectively) (all $P<0.01$, paired- $t$ test). Also, the mean preoperative KOSA-18 scores of the AT alone group and the AT with CAT group (mean \pm SD; $66.8 \pm 20.1$ and $72.0 \pm 19.3$, respectively) improved significantly at postoperative 1 month (31.3 \pm 8.1 and $33.9 \pm 13.8$, respectively) and were sustained at postoperative 6 months $(32.7 \pm 11.0$ and $31.7 \pm 13.5$, respectively) (all $P<0.01$, paired $t$-test). However, RM-ANOVAs for the interaction effects (K-ARS X group and KOSA-18 score $\mathrm{X}$ group) showed no significant differences between the 2 groups ( $F=0.62, P=0.54$ and $F=0.64, P=0.53$ ). In summary, both the AT alone group and the AT with CAT group showed consistently significant improvement in behavior and QoL as compared with preoperative status, but there were no differences between these 2 groups.

\section{DISCUSSION}

We investigated longitudinal changes in behavior and QoL after AT in our prospective pediatric cohort by means of serial preand postoperative questionnaires. Also, we subdivided the cohort using various factors that may affect the prognosis and sought to determine factors that are important. We found that K-ARS and KOSA-18 scores plummet immediately after AT and these improvements are sustained up to 6 months. When subdivided by preoperative K-ARS scores, the overt ADHD and ADHD trait groups showed stark improvement at postoperative 1 month but their mean K-ARS scores were still higher than that of the non-ADHD group, whereas the subgroups divided by preoperative KOSA-18 scores showed no differences in the mean KOSA-18 scores at postoperative 1 month. Notably, the changes in behavior and QoL showed no significant differences according to sex, age group, allergic trait, or concurrent CAT.

Immediate and sustained improvement in behavior and QoL Some researchers have evaluated post-AT children at relatively early time points. For instance, De Serres et al. [1] have reported a postoperative QoL survey study with a mean follow-up duration of 35.7 days. Other groups have demonstrated significant improvement in behavioral and emotional difficulties as well as in QoL at 2 to 4.4 months after surgery [15]. By contrast, some researchers have reported relatively long-term follow-up results. For example, Li et al. [16] have demonstrated improvements in behavior 6 months after AT. In another study, improvements in neurocognitive function 6 to 10 months after AT was shown [17]. Other groups have reported significant improvement in neurobehavioral morbidity 1 year after surgery [18]. In the current study, K-ARS and K-OSA scores were much improved at postoperative 1 month and sustained up to 6 months after surgery. However, of 148 patients, only 11 completed follow-up questionnaires at postoperative 1 year and thus postoperative 6 months was chosen as the endpoint of the current study. The low follow-up rate at postoperative 1 year limited the current study and therefore future study with a larger patient group is warranted. Also, relatively low follow-up rate at postoperative 6 months $(48.6 \%)$ may also limit the value of the current prospective cohort. Future study should be performed to further raise the follow-up rate and re-evaluate the value of the current study. 


\section{Considerations on preoperative consultation}

Most of the children in our cohort did not undergo pre- and postoperative polysomnography (PSG). Although we admit that this limits the interpretation of the current study, we may argue that PSG might not be necessary in some pediatric SDB patients with behavior and QoL problems, based on the fact that our subjects that were diagnosed only by caregivers' information and findings on physical examination showed immediate and sustained improvements in behavior and sleep-related QoL. In addition, previous researchers have pointed out that AT may be associated with reduced behavioral morbidity, even among subjects lacking PSG evidence of OSAS [18] and these improvements may not simply be attributable to changes in apneic events [16]. Future studies on the role of PSG in pediatric SDB patients with adenotonsillar hypertrophy, randomized controlled trials comparing PSG groups and non-PSG groups may be of additional help.

Our results indicated that pediatric SDB patients show improvements in behavior and sleep-related QoL after AT regardless of sex, age group, allergic trait, or concurrent CAT. These results are partly in line with a previous report showing uniform improvements in a similar patient group regardless of sex, age, ethnicity, parental education, parental income, or the relative severity of OSAS [15]. Our results and previous reports may be of help to preoperative consultation because pediatric SDB patients with behavioral problems may be benefitted from AT regardless of their demographic factors. Considering that most of the caregivers do not understand symptoms, consequences and treatment options of pediatric SDB secondary to adenotonsillar hypertrophy [19], our findings may play a part in counselling the caregivers before surgery.

While sleep-related QoL showed uniform improvement without any significant differences at postoperative 1 month, children with abnormal preoperative behavior displayed improved but still significantly higher K-ARS scores than children without preoperative abnormal behavior. In addition, although only 6 children could be evaluated, the mean K-ARS score of the overt ADHD group at 6 months, which was in the range of "ADHDtrait", was significantly higher than that at 1 month. In other words, sleep-related QoL is normalized regardless of preoperative impairment whereas behavioral problems may not be completely solved in children with substantial preoperative behavioral problems. In this regard, long-term follow-up with repeated behavioral evaluation may be mandatory in children with overt ADHD or ADHD-trait.

One important discrepancy between our study and a previous report should be discussed. While our results shows no difference with regard to combined allergic trait or concurrent CAT, Cheng et al. [20] showed that AT with concurrent turbinoplasty achieves favorable outcomes regarding QoL in pediatric OSAS with allergic rhinitis. This discrepancy may be attributed to the differences in inclusion criteria and method of inferior turbino- plasty. That is, Cheng et al. [20] collected subjects with documented intractable allergic rhinitis and congested inferior turbinate for at least 3 months, while our subjects' chief complaints were mainly snoring and related problems, not allergy or nasal obstruction. Therefore, Cheng et al. [20] may have collected severer cases than ours and this may have resulted in the discrepancy. Future studies with stricter inclusion criteria and large number of cases should be performed to clarify this discrepancy.

The current prospective cohort study demonstrated immediate improvements in behavior and sleep-related QoL after AT in children with SDB and these improvements were sustained up to 6 months without any significant change. Also, these improvements in behavior and sleep-related QoL were uniformly significant regardless of preoperative symptom severity. Moreover, improvements in behavior and QoL are expected regardless of sex, age group, allergic trait, or concurrent CAT. Although future studies are needed to prove causality, these findings may be of crucial help to our colleague physicians in counseling pediatric SDB patients.

\section{CONFLICT OF INTEREST}

No potential conflict of interest relevant to this article was reported.

\section{REFERENCES}

1. De Serres LM, Derkay C, Sie K, Biavati M, Jones J, Tunkel D, et al. Impact of adenotonsillectomy on quality of life in children with obstructive sleep disorders. Arch Otolaryngol Head Neck Surg. 2002 May;128(5):489-96.

2. Spruyt K, Gozal D. Sleep disturbances in children with attentiondeficit/hyperactivity disorder. Expert Rev Neurother. 2011 Apr;11 (4):565-77.

3. Huang YS, Guilleminault C, Li HY, Yang CM, Wu YY, Chen NH. Attention-deficit/hyperactivity disorder with obstructive sleep apnea: a treatment outcome study. Sleep Med. 2007 Jan;8(1):18-30.

4. Bhattacharjee R, Kheirandish-Gozal L, Spruyt K, Mitchell RB, Promchiarak J, Simakajornboon N, et al. Adenotonsillectomy outcomes in treatment of obstructive sleep apnea in children: a multicenter retrospective study. Am J Respir Crit Care Med. 2010 Sep; 182(5):676-83.

5. Wei JL, Bond J, Mayo MS, Smith HJ, Reese M, Weatherly RA. Improved behavior and sleep after adenotonsillectomy in children with sleep-disordered breathing: long-term follow-up. Arch Otolaryngol Head Neck Surg. 2009 Jul;135(7):642-6.

6. Baldassari CM, Mitchell RB, Schubert C, Rudnick EF. Pediatric obstructive sleep apnea and quality of life: a meta-analysis. Otolaryngol Head Neck Surg. 2008 Mar;138(3):265-73.

7. Mitchell RB, Kelly J. Long-term changes in behavior after adenotonsillectomy for obstructive sleep apnea syndrome in children. Otolaryngol Head Neck Surg. 2006 Mar;134(3):374-8.

8. McColley SA, Carroll JL, Curtis S, Loughlin GM, Sampson HA. High prevalence of allergic sensitization in children with habitual snoring and obstructive sleep apnea. Chest. 1997 Jan;111(1):170-3. 
9. Yoo HJ, Cho SC, Ha J, Yune SK, Kim SJ, Hwang J, et al. Attention deficit hyperactivity symptoms and internet addiction. Psychiatry Clin Neurosci. 2004 Oct;58(5):487-94.

10. DuPaul GJ, Power TJ, Anastopoulos AD, Reid R. ADHD rating scaleIV: checklists, norms, and clinical interpretation. New York: Guilford Press; 1998.

11. Katic A, Dirks B, Babcock T, Scheckner B, Adeyi B, Richards C, et al. Treatment outcomes with lisdexamfetamine dimesylate in children who have attention-deficit/hyperactivity disorder with emotional control impairments. J Child Adolesc Psychopharmacol. 2013 Aug; 23(6):386-93.

12. Hwang SH, Guilleminault C, Park CS, Kim TW, Hong SC. Usefulness of adenotonsillar size for prediction of severity of obstructive sleep apnea and flow limitation. Otolaryngol Head Neck Surg. 2013 Aug; 149(2):326-34.

13. Fischer Y, Rettinger G, Dorn M. Long term change in quality of life after adenotonsillectomy for pediatric obstructive sleep disorders. Laryngorhinootologie. 2006 Nov;85(11):809-18.

14. Franco RA Jr, Rosenfeld RM, Rao M. First place: resident clinical science award 1999. Quality of life for children with obstructive sleep apnea. Otolaryngol Head Neck Surg. 2000 Jul;123(1 Pt 1):9-16.
15. Mitchell RB, Kelly J. Child behavior after adenotonsillectomy for obstructive sleep apnea syndrome. Laryngoscope. 2005 Nov;115 (11):2051-5.

16. Li HY, Huang YS, Chen NH, Fang TJ, Lee LA. Impact of adenotonsillectomy on behavior in children with sleep-disordered breathing. Laryngoscope. 2006 Jul;116(7):1142-7.

17. Friedman BC, Hendeles-Amitai A, Kozminsky E, Leiberman A, Friger M, Tarasiuk A, et al. Adenotonsillectomy improves neurocognitive function in children with obstructive sleep apnea syndrome. Sleep. 2003 Dec;26(8):999-1005.

18. Dillon JE, Blunden S, Ruzicka DL, Guire KE, Champine D, Weatherly RA, et al. DSM-IV diagnoses and obstructive sleep apnea in children before and 1 year after adenotonsillectomy. J Am Acad Child Adolesc Psychiatry. 2007 Nov;46(11):1425-36.

19. Strocker AM, Shapiro NL. Parental understanding and attitudes of pediatric obstructive sleep apnea and adenotonsillectomy. Int J Pediatr Otorhinolaryngol. 2007 Nov;71(11):1709-15.

20. Cheng PW, Fang KM, Su HW, Huang TW. Improved objective outcomes and quality of life after adenotonsillectomy with inferior turbinate reduction in pediatric obstructive sleep apnea with inferior turbinate hypertrophy. Laryngoscope. 2012 Dec;122(12):2850-4. 